\title{
6 Agriculture, food, drinks and tobacco
}

\section{Land use and crop areas ${ }^{1}$}

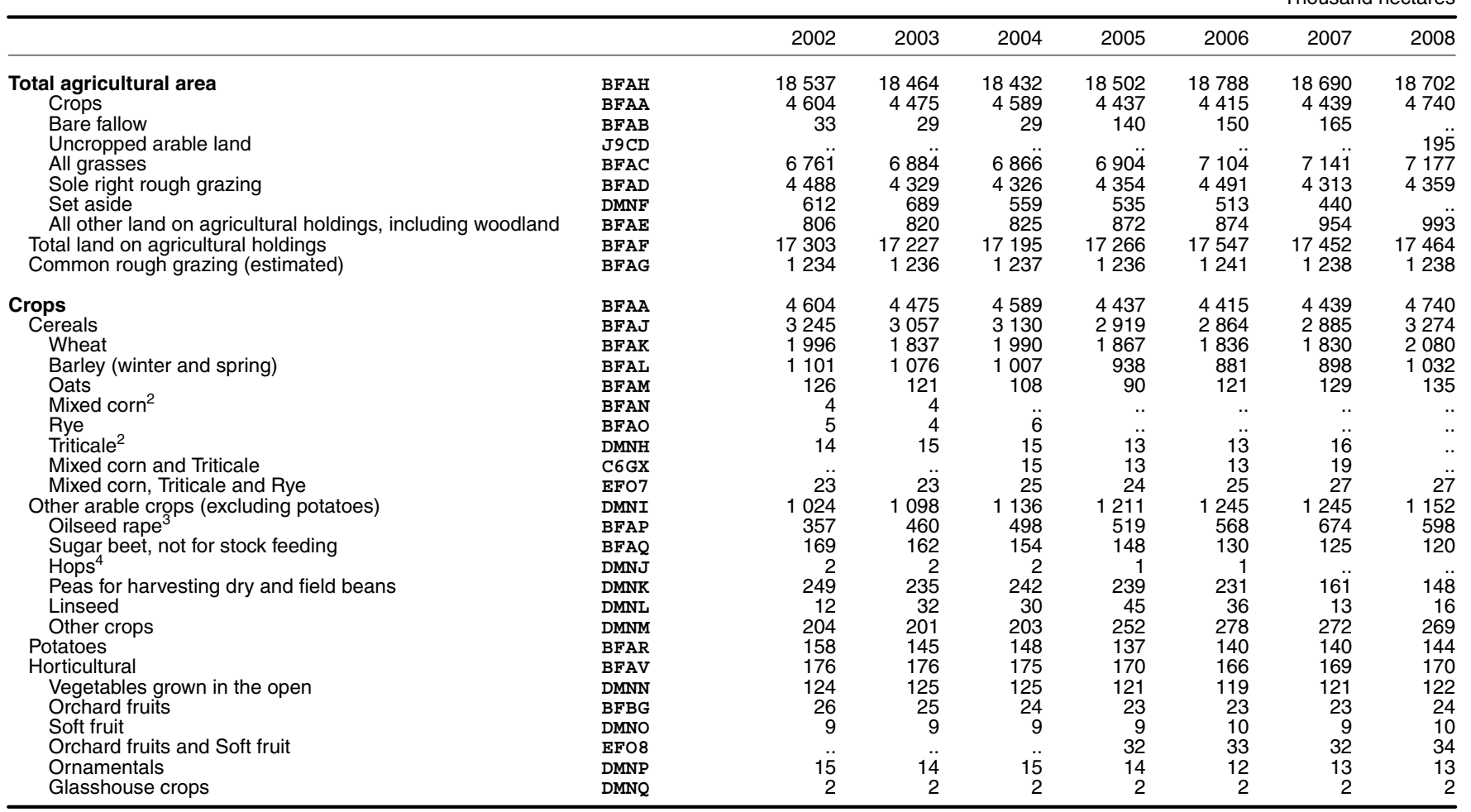

1 Figures include estimates for minor holdings. For further information

2 From 2004 onwards data for Mixed corn and Triticale amalgamated.

refer to Section 6 of the Annual Supplement in the January edition of 3 Area grown not on set-aside land.

Monthly Digest.

4 From 2007 hops are included in "other crops" category.

Source: Department for Environment, Food and Rural Affairs: 01904455095

\section{2 Crops: yields and production ${ }^{1}$}

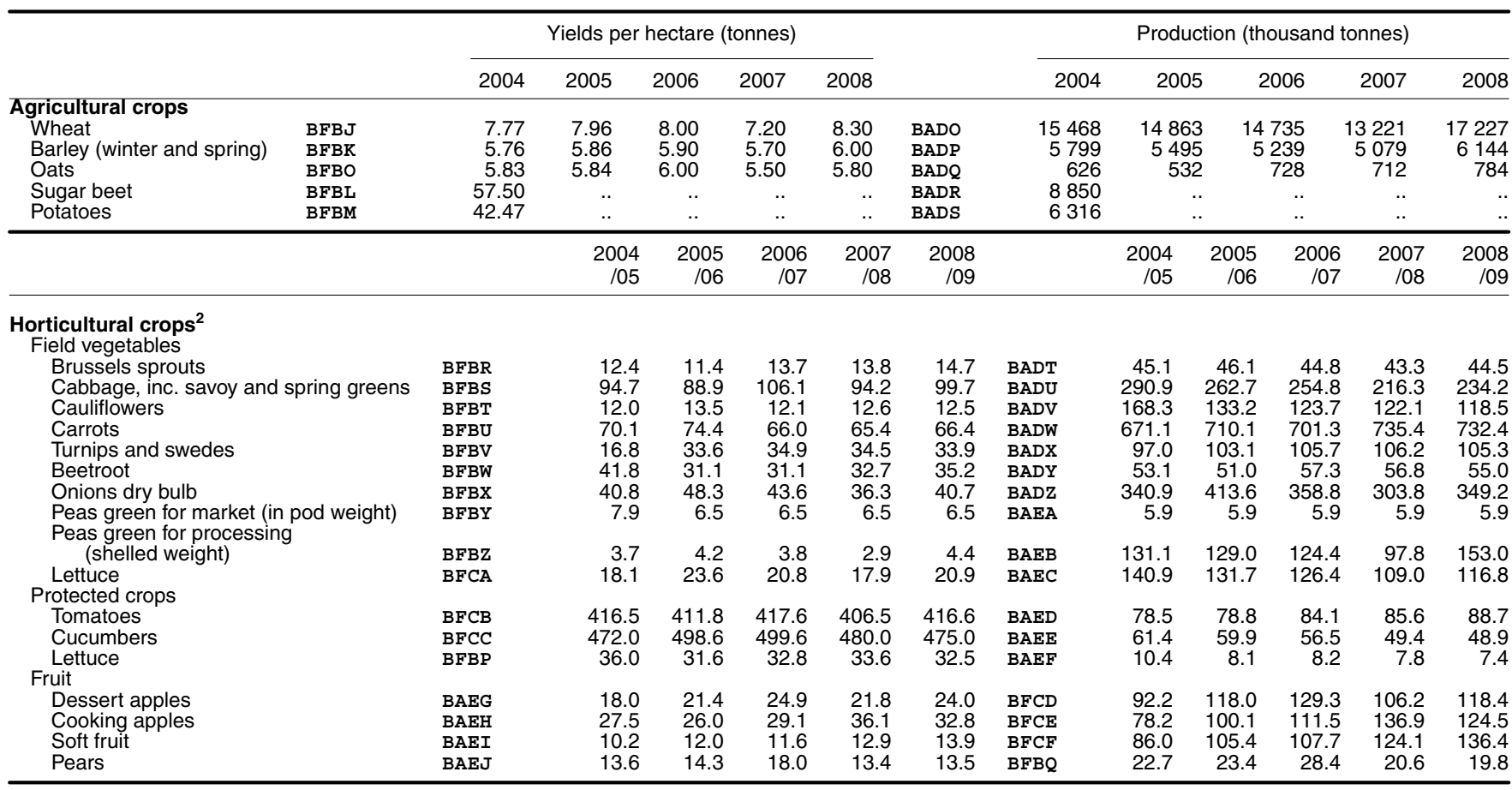

1 For further information, refer to Section 6 of the Annual Supplement in the January edition of Monthly Digest.

2 Yield data are marketed yield and production data are home production marketed. 


\section{$6.3^{\text {Livesocok' }}$}

\begin{tabular}{|c|c|c|c|c|c|c|c|c|c|c|c|c|c|}
\hline & & $\begin{array}{r}2002 \\
\text { Dec }\end{array}$ & $\begin{array}{r}2003 \\
\text { Jun }\end{array}$ & $\begin{array}{r}2003 \\
\text { Dec }\end{array}$ & $\begin{array}{r}2004 \\
\text { Jun }\end{array}$ & $\begin{array}{r}2004 \\
\text { Dec }\end{array}$ & $\begin{array}{r}2005 \\
\text { Jun }\end{array}$ & $\begin{array}{r}2005 \\
\text { Dec }\end{array}$ & $\begin{array}{r}2006 \\
\text { Jun }\end{array}$ & $\begin{array}{r}2006 \\
\text { Dec }\end{array}$ & $\begin{array}{r}2007 \\
\text { Jun }\end{array}$ & $\begin{array}{r}2007 \\
\text { Dec }\end{array}$ & $\begin{array}{r}2008 \\
\text { Jun }\end{array}$ \\
\hline $\begin{array}{l}\text { Total cattle and calves }{ }^{2} \\
\text { Dairy cows } \\
\text { Beef cows } \\
\text { Heifers in calf }\end{array}$ & $\begin{array}{l}\text { BFCG } \\
\text { BFCH } \\
\text { BFCI } \\
\text { BFCJ }\end{array}$ & $\begin{array}{r}10381 \\
2229 \\
1694 \\
684\end{array}$ & $\begin{array}{r}10508 \\
2191 \\
1698 \\
679\end{array}$ & $\begin{array}{r}10519 \\
2207 \\
1702 \\
678\end{array}$ & $\begin{array}{r}10588 \\
2129 \\
1736 \\
690\end{array}$ & $\begin{array}{r}10425 \\
2152 \\
1733 \\
680\end{array}$ & $\begin{array}{r}10392 \\
2063 \\
1762 \\
638\end{array}$ & $\begin{array}{r}10160 \\
2060 \\
1667 \\
697\end{array}$ & $\begin{array}{r}10270 \\
2066 \\
1733 \\
645\end{array}$ & $\begin{array}{r}10010 \\
2024 \\
1662 \\
670\end{array}$ & $\begin{array}{r}10304 \\
1954 \\
1698\end{array}$ & $\begin{array}{r}10075 \\
1977 \\
1663 \\
. .\end{array}$ & $\begin{array}{r}10107 \\
1909 \\
1670\end{array}$ \\
\hline $\begin{array}{l}\text { Total sheep and lambs } \\
\text { Ewes and shearlings } \\
\text { Lambs under one year old }\end{array}$ & $\begin{array}{l}\text { BFCM } \\
\text { CKUQ } \\
\text { BFCP }\end{array}$ & $\begin{array}{r}24898 \\
16469 \\
7233\end{array}$ & $\begin{array}{l}35812 \\
17580 \\
17322\end{array}$ & $\begin{array}{rl}24 & 572 \\
16337 \\
7078\end{array}$ & $\begin{array}{l}35817 \\
17630 \\
17238\end{array}$ & $\begin{array}{r}24688 \\
16308 \\
7067\end{array}$ & $\begin{array}{l}35416 \\
16935 \\
17488\end{array}$ & $\begin{array}{r}23933 \\
15601 \\
7146\end{array}$ & $\begin{array}{l}34722 \\
16637 \\
17058\end{array}$ & $\begin{array}{r}23428 \\
14164 \\
7300\end{array}$ & $\begin{array}{l}33946 \\
16064 \\
16855\end{array}$ & $\begin{array}{r}23676 \\
14841 \\
7663\end{array}$ & $\begin{array}{l}33131 \\
15616 \\
16574\end{array}$ \\
\hline $\begin{array}{l}\text { Total pigs } \\
\text { Sows in pig and other } \\
\text { sows for breeding } \\
\text { Gilts in pig }\end{array}$ & $\begin{array}{l}\text { CKUU } \\
\text { CKUR }\end{array}$ & $\begin{array}{r}446 \\
74\end{array}$ & $\begin{array}{r}442 \\
73\end{array}$ & $\begin{array}{r}444 \\
70\end{array}$ & $\begin{array}{r}449 \\
66\end{array}$ & $\begin{array}{r}413 \\
63\end{array}$ & $\begin{array}{r}404 \\
67\end{array}$ & $\begin{array}{r}376 \\
65\end{array}$ & $\begin{array}{r}401 \\
67\end{array}$ & $\begin{array}{r}387 \\
62\end{array}$ & $\begin{array}{r}398 \\
57\end{array}$ & $\begin{array}{r}383 \\
54\end{array}$ & $\begin{array}{r}365 \\
55\end{array}$ \\
\hline
\end{tabular}

1 Figures include estimates for minor holdings. For further details refer to section 6 of the Annual Supplement in the January edition of Monthly Digest.

2 In 2007 the cattle figures were sourced from the Cattle Tracing System (CTS) in England and Wales, the equivalent APHIS system in Northern Ireland and survey data in Scotland and are therefore not directly comparable with earlier years. To see comparable data for 2005-2007 please go to http://st atistics.defra.gov.uk/esg/statnot/june_uk.pdf

\subsection{Animals slaughterese and meat produccod \\ Monthly averages, totals for four or five week periods and quarters}

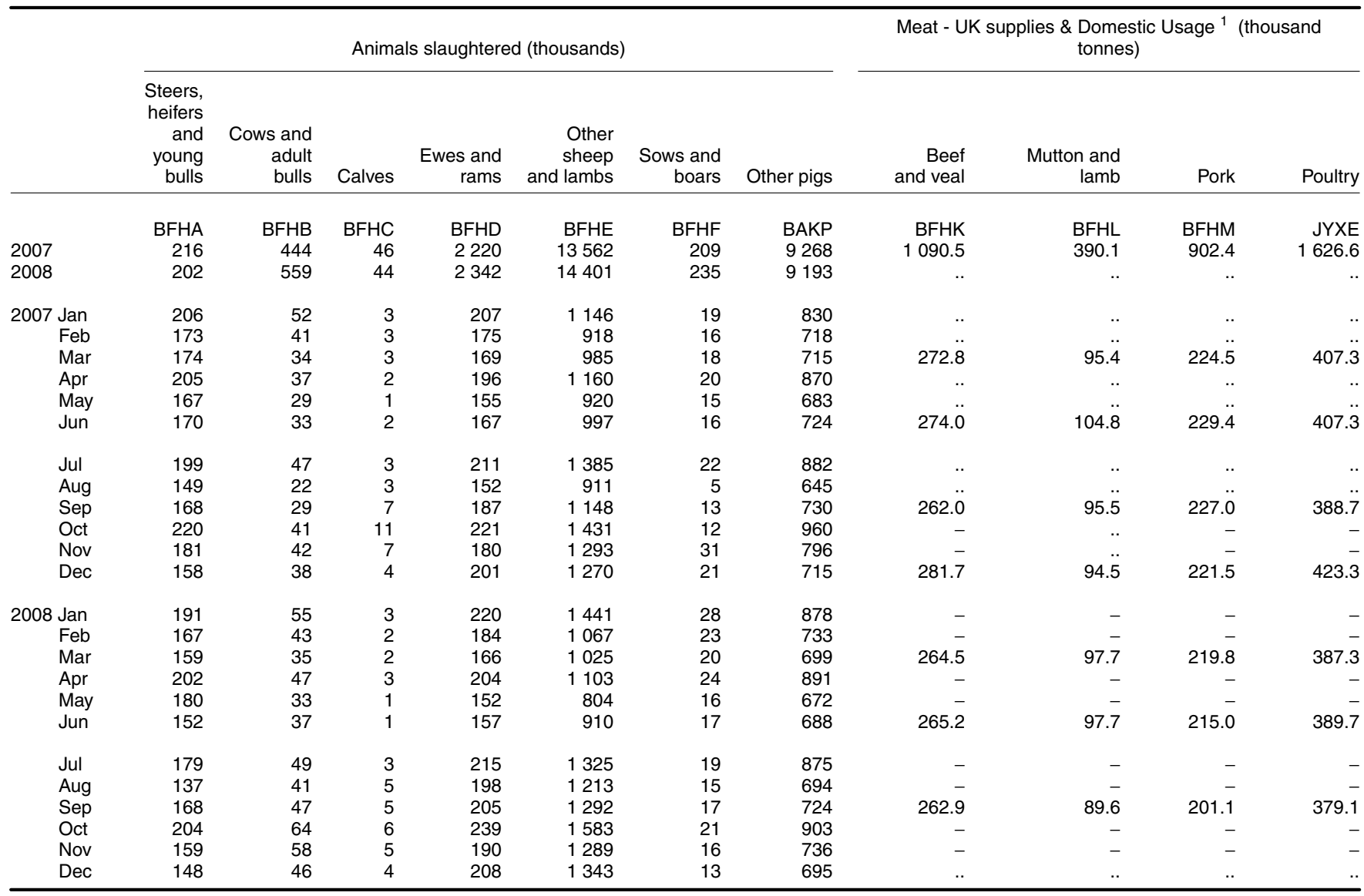

1 From January 2007, 'Meat - UK supplies \& Domestic Usage' are shown as quarters and the data are provisional.

Source: Department for Environment, Food and Rural Affairs: 01904455097 
6 Cereals and cereal products

. Monthly averages or totals for four or five week periods. Stocks refer to the end of the period

Thousand tonnes

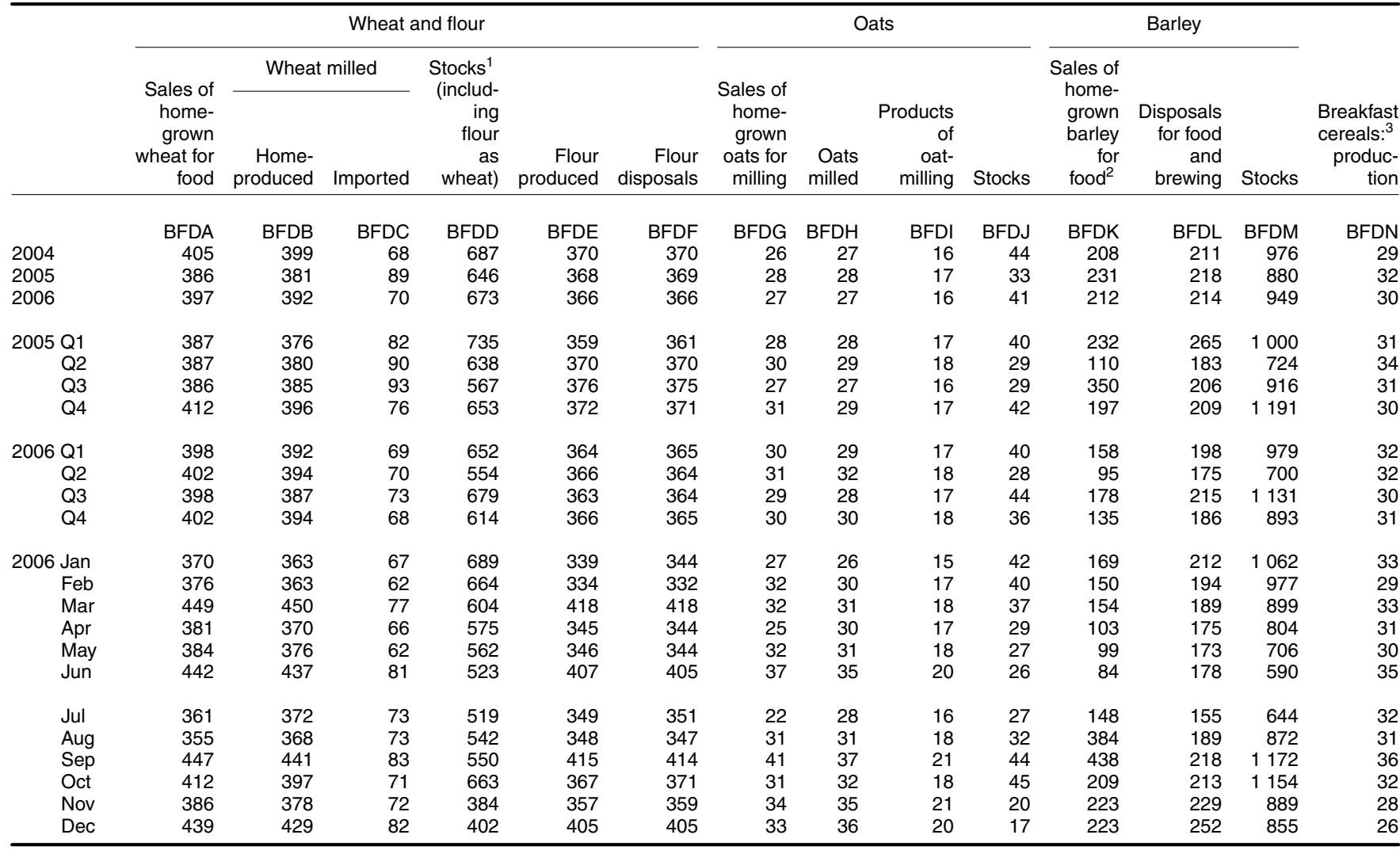

1 Stocks held by wheat millers, feed compounders, cereal breakfast food Source: Department for Environment, Food and Rural Affairs: 01904455076 manufacturers, brewers, maltsters and distillers, merchants and dealers.

2 Sales of UK grown barley to brewers, maltsters and distillers.

3 Other than oatmeal and oatmeal flakes.

\subsection{Production of compound feedingstuffs}

Thousand tonnes

\begin{tabular}{|c|c|c|c|c|c|c|c|}
\hline & $\begin{array}{r}\text { Cattle and calf } \\
\text { feed }\end{array}$ & Pig feed & Poultry feed & Sheep feed & Horse feed & Other compounds & Total \\
\hline & BFFB & BFFD & BFFE & JJ8R & JJ8S & BFFF & BFFA \\
\hline Mar & 384.4 & 139.3 & 248.2 & 153.6 & 19.9 & 21.5 & 966.9 \\
\hline Apr & 305.0 & 123.2 & 217.4 & 79.6 & 14.8 & 22.5 & 762.4 \\
\hline May & 239.2 & 126.8 & 230.7 & 32.1 & 13.4 & 26.5 & 668.7 \\
\hline Jun & 249.1 & 145.0 & 269.9 & 25.1 & 11.9 & 33.0 & 734.0 \\
\hline Jul & 268.8 & 133.0 & 259.2 & 21.1 & 12.5 & 34.0 & 728.6 \\
\hline Aug & 294.2 & 134.5 & 251.8 & 25.1 & 13.0 & 36.2 & 754.7 \\
\hline Sep & 322.6 & 147.1 & 253.8 & 25.0 & 15.7 & 36.3 & 800.6 \\
\hline Oct & 332.1 & 137.7 & 237.1 & 24.5 & 15.9 & 30.2 & 777.6 \\
\hline Nov & 341.3 & 132.2 & 237.4 & 31.8 & 18.5 & 25.9 & 787.2 \\
\hline Dec & 395.2 & 144.2 & 253.8 & 51.8 & 20.6 & 25.3 & 890.9 \\
\hline 2008 Jan & 352.8 & 123.6 & 222.5 & 84.5 & 20.2 & 21.8 & 825.5 \\
\hline Feb & 353.5 & 116.2 & 215.7 & 109.3 & 18.7 & 22.7 & 836.1 \\
\hline Mar & 392.1 & 132.5 & 248.2 & 142.3 & 20.3 & 24.4 & 959.8 \\
\hline May & 245.1 & 114.9 & 231.9 & 29.9 & 12.8 & 27.4 & 662.0 \\
\hline Jun & 250.6 & 125.9 & 270.7 & 23.1 & 11.5 & 25.6 & 707.4 \\
\hline Jul & 256.8 & 119.0 & 265.2 & 18.6 & 11.9 & 28.2 & 699.6 \\
\hline Aug & 262.9 & 115.1 & 240.1 & 17.4 & 11.6 & 31.9 & 679.0 \\
\hline Sep & 319.9 & 130.7 & 263.8 & 26.0 & 16.9 & 38.3 & 795.5 \\
\hline Oct & 333.9 & 118.7 & 238.8 & 31.6 & 15.8 & 32.9 & 771.9 \\
\hline Nov & 321.2 & 112.8 & 227.5 & 43.7 & 16.7 & 27.1 & 749.0 \\
\hline Dec & 382.9 & 128.9 & 251.4 & 63.6 & 21.6 & 27.5 & 875.9 \\
\hline 2009 Jan & 327.3 & 104.7 & 210.8 & 91.3 & 17.8 & 24.9 & 776.8 \\
\hline Feb & 315.5 & 101.8 & 198.7 & 112.8 & 17.2 & 23.8 & 769.9 \\
\hline Mar & 369.5 & 116.9 & 242.6 & 142.1 & 19.1 & 18.7 & 908.9 \\
\hline Apr & 295.4 & 106.4 & 221.2 & 82.6 & 15.7 & 24.5 & 745.8 \\
\hline May & 249.4 & 105.4 & 229.8 & 36.4 & 11.8 & 27.8 & 660.5 \\
\hline
\end{tabular}


Thousand tonnes

\begin{tabular}{|c|c|c|c|c|c|c|c|c|c|}
\hline & \multicolumn{4}{|c|}{ Potatoes } & \multicolumn{4}{|c|}{ Sugar (as refined) } & \multirow[b]{3}{*}{$\begin{array}{r}\text { Glucose } \\
\text { production }\end{array}$} \\
\hline & \multicolumn{3}{|c|}{$\begin{array}{l}\text { Movement into human consumption in the United } \\
\text { Kingdom }\end{array}$} & \multirow[b]{2}{*}{ Stocks ${ }^{4}$} & \multirow{2}{*}{$\begin{array}{r}\text { Production from } \\
\text { home- grown } \\
\text { sugar beet }\end{array}$} & \multicolumn{2}{|c|}{ Disposals } & \multirow[b]{2}{*}{ Stocks } & \\
\hline & From home crop & Imports $^{2,3}$ & Exports $^{3}$ & & & $\begin{array}{c}\text { Total New } \\
\text { Supply }\end{array}$ & $\begin{array}{l}\text { For food in the } \\
\text { United Kingdom }\end{array}$ & & \\
\hline & BFGA & BFGB & BFGC & BFGD & BFGF & BFGG & BFGH & BFGI & BFGK \\
\hline 2002 & 417 & 150 & 30 & 3386 & 1430.0 & 2197.0 & .. & .. & 60.6 \\
\hline 2003 & 400 & 142 & 31 & 2915 & 1368.0 & 1800.0 & .. & .. & 61.2 \\
\hline 2004 & 391 & 148 & 26 & 2820 & 1390.0 & 1874.0 & .. & .. & 61.4 \\
\hline 2005 & 361 & 124 & 27 & 2509 & 1300.0 & 1859.0 & .. & .. & 57.9 \\
\hline 2006 & 355 & .. & .. & .. & .. & .. & .. & .. & 46.7 \\
\hline 2006 Feb & 343 & .. & .. & .. & 0.1 & 144.1 & 143.4 & 731.9 & 44.5 \\
\hline Mar & 377 & .. & .. & .. & 2.9 & 151.6 & 151.3 & 627.5 & 50.7 \\
\hline Apr & 301 & .. & .. & .. & 3.3 & 165.0 & 164.4 & 525.6 & 47.4 \\
\hline May & 305 & .. & .. & .. & 3.1 & 151.9 & 151.3 & 428.9 & 49.7 \\
\hline Jun & 281 & .. & .. & .. & .. & .. & .. & .. & 44.8 \\
\hline Jul & 277 & .. & .. & .. & .. & .. & .. & .. & 47.6 \\
\hline Aug & 390 & .. & .. & .. & .. & .. & .. & .. & 47.8 \\
\hline Sep & 444 & .. & .. & .. & .. & .. & .. & .. & 45.7 \\
\hline Oct & 521 & .. & .. & .. & .. & .. & .. & .. & 47.7 \\
\hline Nov & 420 & .. & .. & .. & .. & .. & .. & .. & 47.0 \\
\hline Dec & 368 & .. & .. & .. & .. & .. & .. & .. & 41.2 \\
\hline 2007 Jan &.. & .. & .. & .. & .. & .. & .. & .. & 42.2 \\
\hline Feb &.. & .. & .. & .. & .. & .. & .. & .. & 43.0 \\
\hline Mar & ... & .. & .. & .. & .. & .. & .. & .. & 49.3 \\
\hline
\end{tabular}

*Note: The annual figures for sugar are calendar year totals, rather than $12 \quad 5$ Total New supply (including imports) for use by UK food and other industries month averages. (including sugar used in the chemical industry). For further information:

1 For further information refer to Section 6 of the Annual Supplement in the http://statistics.defra.gov.uk/esg/publications/auk/2005/5-7.xls

January edition of Monthly Digest.

2 Includes Channel Isles exports to Great Britain.

3 Trade data provided by British Potato Council and Dept. of Agriculture and Sources: Department for Environment, Food and Rural Affairs 01904455067 (glucose); 02072383279 (sugar); Rural Development in Northern Ireland. Figures currently unavailable for 01904455068 (potatoes)

4 Estimate of end - December stocks based on Potato Marketing returns.

\subsection{Production of pork, bacon and ham, beef and veal, mutton and lamb, poultrymeat ${ }^{1}$}

\begin{tabular}{|c|c|c|c|c|c|c|}
\hline & $\begin{array}{c}\text { Pork }^{2} \\
\text { Home production }\end{array}$ & $\begin{array}{c}\text { Bacon and ham } \\
\text { Home cured production }\end{array}$ & $\begin{array}{l}\text { Beef and veal }^{2} \\
\text { Home production }\end{array}$ & $\begin{array}{l}\text { Mutton and lamb } \\
\text { Home production }\end{array}$ & $\begin{array}{c}\text { Poultry meat } \\
\text { Home cured production }\end{array}$ & $\begin{array}{c}\text { All Meat } \\
\text { Home production }\end{array}$ \\
\hline 2002 & 595.0 & 231.0 & 694.3 & 307.3 & 1556.7 & 3384.3 \\
\hline 2003 & 541.8 & 236.6 & 703.2 & 309.8 & 1578.1 & 3369.5 \\
\hline 2004 & 547.2 & 235.1 & 723.6 & 319.2 & 1571.1 & З 396.2 \\
\hline 2005 & 543.4 & 239.8 & 764.8 & 336.7 & 1585.0 & 3469.8 \\
\hline $2007^{3}$ & 599.2 & 196.6 & 888.5 & 329.5 & 1459.2 & 3473.0 \\
\hline 2008 & 602.5 & 183.7 & 866.0 & 331.6 & 1429.7 & 3413.7 \\
\hline 2005 Q2 & 133.3 & 60.6 & 191.3 & 70.6 & 388.7 & 844.6 \\
\hline Q3 & 135.2 & 57.6 & 190.9 & 89.1 & 390.3 & 863.1 \\
\hline Q4 & 137.2 & 64.5 & 204.3 & 97.1 & 401.3 & 904.4 \\
\hline $2006 \mathrm{Q}^{3}$ & 134.3 & 56.0 & 206.8 & 83.5 & 377.9 & 857.5 \\
\hline $\mathrm{Q}^{3}$ & 126.9 & 58.7 & 207.1 & 69.5 & 384.5 & 846.7 \\
\hline $\mathrm{Q} 4^{3}$ & 147.8 & 58.8 & 228.8 & 92.6 & 382.1 & 910.1 \\
\hline $2007 \mathrm{Q} 1^{3}$ & 149.3 & 48.5 & 228.0 & 75.9 & 366.7 & 868.3 \\
\hline $\mathrm{Q}^{3}$ & 146.1 & 47.8 & 220.3 & 76.4 & 369.0 & 859.6 \\
\hline $\mathrm{Q3}^{3}$ & 141.6 & 49.3 & 209.6 & 81.9 & 352.2 & 834.6 \\
\hline $\mathrm{Q} 4^{3}$ & 162.2 & 51.0 & 230.6 & 95.3 & 371.4 & 910.5 \\
\hline 2008 Q1 & 154.8 & 45.0 & 221.7 & 83.8 & 353.2 & 858.6 \\
\hline Q2 & 145.4 & 45.0 & 214.3 & 68.3 & 356.8 & 829.7 \\
\hline Q3 & 150.5 & 45.0 & 207.5 & 86.8 & 354.0 & 843.8 \\
\hline Q4 & 151.8 & 48.7 & 222.5 & 92.7 & 365.8 & 881.6 \\
\hline 2009 Q1 & 147.6 & 42.5 & 210.4 & 72.3 & 354.8 & 827.6 \\
\hline
\end{tabular}

1 Does not include meat offals or trade in preserved or manufactured meat 3 Figures have been rounded individually and totals will not necessarily agree products (e.g. canned meat).

2 Includes an estimate of the dressed carcase weight of live exports other with the addition of separate figures. 


\begin{tabular}{|c|c|c|c|c|c|c|c|c|c|c|c|}
\hline & \multirow{3}{*}{$\begin{array}{r}\text { Fresh and } \\
\text { frozen fish: } \\
\text { UK landings }\end{array}$} & \multicolumn{3}{|c|}{ Oilseeds and nuts } & \multirow{2}{*}{\multicolumn{2}{|c|}{$\begin{array}{c}\text { Vegetable oil } \\
\text { Crude oil } \\
\text { equivalent }\end{array}$}} & \multirow{2}{*}{\multicolumn{2}{|c|}{$\begin{array}{c}\text { Marine oil } \\
\begin{array}{c}\text { Crude oil } \\
\text { equivalent }\end{array}\end{array}$}} & \multirow{3}{*}{$\begin{array}{r}\text { Margar- } \\
\text { ine: } \\
\text { produc- } \\
\text { tion }\end{array}$} & \multirow{3}{*}{$\begin{array}{r}\text { Solid } \\
\text { cooking } \\
\text { fat }\end{array}$} & \multirow{3}{*}{$\begin{array}{r}\text { Other } \\
\text { table } \\
\text { spreads }\end{array}$} \\
\hline & & \multirow[b]{2}{*}{ Crushed } & & \multirow{2}{*}{$\begin{array}{r}\text { Stocks }^{5} \text { : } \\
\text { crude oil } \\
\text { equiv- } \\
\text { alent }\end{array}$} & & & & & & & \\
\hline & & & produced & & Disposals $^{1}$ & Stocks 2,5 & Usage $^{3}$ & Stocks $s^{4,5}$ & & & \\
\hline & BFJA & BFJE & BFJF & BFJG & BFJJ & BFJK & BFJL & BFJM & BFJN & BFJO & BFJP \\
\hline $2003^{6}$ & .. & 2210.3 & 768.9 & 13.2 & 2213.7 & 86.5 & 2.1 & - & 135.9 & 130.9 & 305.9 \\
\hline 2004 & .. & 2128.2 & 747.5 & 23.1 & 2058.2 & 170.8 & 1.7 & .. & 114.5 & 130.5 & 316.5 \\
\hline 2005 &.. & .. & .. & .. & 2043.6 & 166.9 & 1.2 & .. & 115.9 & 117.0 & 292.7 \\
\hline 2006 & 22.8 & .. & .. & .. & .. & .. & .. & .. & .. & .. & .. \\
\hline 2007 & 28.5 & .. & .. & .. & .. & .. & .. & .. & .. & .. & .. \\
\hline 2008 Feb & 31.5 & .. & .. & .. & .. & .. & .. & .. & .. & .. & .. \\
\hline Mar & 19.8 & .. & .. & .. & .. & .. & .. & .. & .. & .. & .. \\
\hline Apr & 11.6 & .. & .. & .. & .. & .. & .. & .. & .. & .. & .. \\
\hline May & 9.8 & .. & .. & .. & .. & .. & .. & .. & .. & .. & .. \\
\hline Jun & 10.6 & .. & .. & .. & .. & .. & .. & .. & .. & .. & .. \\
\hline Jul & 12.3 & .. & .. & .. & .. & .. & .. & .. & .. & .. & .. \\
\hline Sep & 18.3 & .. & .. & .. & .. & .. & .. & .. & .. & .. & .. \\
\hline Oct & 39.9 & .. & .. & .. & .. & .. & .. & .. & .. & .. & .. \\
\hline Nov & 11.7 & .. & .. & .. & .. & .. & .. & .. & .. & .. & .. \\
\hline Dec & 8.7 & .. & .. & .. & .. & .. & .. & .. & .. & .. & .. \\
\hline 2009 Jan & 59.4 & .. & .. & .. & .. & .. & .. & .. & .. & .. & .. \\
\hline Feb & 32.9 & .. & .. & .. & .. & .. & .. & .. & .. & .. & .. \\
\hline Mar & 12.0 & .. & .. & .. & .. & .. & .. & .. & .. & .. & .. \\
\hline Apr & 10.9 & .. & .. & .. & .. & .. & .. & .. & .. & .. & .. \\
\hline
\end{tabular}

1 This series contains revisions following the incorporation of revised trade figures.

2 Comprising stocks of crude and refined oils held by seed crushers, oil refiners and manufacturers of margarine, solid cooking fat and other table spreads.

3 For the manufacture of margarine, solid cooking fat and other table spreads only.
4 Including quantities held by hardeners and refiners of oil and manufacturers of margarine.

ures for oil seeds and nuts,

6 Figures for 2002-2003 are shown in actual annuals totals.

Sources: Department for Environment, Food and Rural Affairs 02072385913 (fish landings); 01904455061 (oils and fats)

\subsection{Milk and milk productsis}

\begin{tabular}{|c|c|c|c|c|c|c|c|c|}
\hline & \multicolumn{4}{|c|}{ Million litres } & \multicolumn{4}{|c|}{ Thousand tonnes } \\
\hline & \multicolumn{4}{|c|}{ Disposals of raw milk } & \multicolumn{4}{|c|}{ Wholesale production ${ }^{3}$} \\
\hline & Liquid milk ${ }^{2}$ & $\begin{array}{l}\text { For manu- } \\
\text { facture }\end{array}$ & Exports & $\begin{array}{r}\text { Stock change } \\
\& \text { wastage }\end{array}$ & Condensed milk ${ }^{4}$ & Milk powders ${ }^{4,5}$ & Butter & Cheese \\
\hline $\begin{array}{l}2004 \\
2005 \\
2006 \\
2007 \\
2008\end{array}$ & $\begin{array}{rr} & \text { IFX4 } \\
6 & 693.3 \\
6 & 651.7 \\
6 & 736.0 \\
6 & 723.7 \\
6 & 682.7\end{array}$ & $\begin{array}{lr} & \text { IFX5 } \\
6 & 827.5 \\
6 & 568.4 \\
6 & 343.3 \\
6 & 085.1 \\
5 & 843.5\end{array}$ & $\begin{array}{r}\text { IFX6 } \\
434.3 \\
623.9 \\
610.4 \\
537.6 \\
559.2\end{array}$ & $\begin{array}{r}\text { IFX7 } \\
3.5 \\
14.6 \\
37.6 \\
126.8 \\
91.2\end{array}$ & $\begin{array}{r}\text { IFX8 } \\
161.2 \\
142.7 \\
113.3 \\
109.2 \\
109.9\end{array}$ & $\begin{array}{r}\text { IFX9 } \\
80.1 \\
52.4 \\
114.1 \\
117.0 \\
94.3\end{array}$ & $\begin{array}{r}\text { IFY2 } \\
121.7 \\
130.0 \\
117.1 \\
120.4 \\
111.0\end{array}$ & $\begin{array}{r}\text { IFY3 } \\
351.3 \\
384.2 \\
393.4 \\
372.6 \\
382.1\end{array}$ \\
\hline $\begin{array}{c}2008 \text { Feb } \\
\text { Mar } \\
\text { Apr } \\
\text { May } \\
\text { Jun }\end{array}$ & $\begin{array}{l}553.0 \\
571.6 \\
549.7 \\
555.8 \\
519.0\end{array}$ & $\begin{array}{l}459.9 \\
524.5 \\
541.4 \\
617.4 \\
558.1\end{array}$ & $\begin{array}{l}41.0 \\
46.1 \\
46.2 \\
51.1 \\
42.4\end{array}$ & $\begin{array}{r}4.5 \\
9.4 \\
16.9 \\
18.1 \\
22.6\end{array}$ & $\begin{array}{r}25 . \overline{7} \\
- \\
\overline{-} \\
29 . \overline{9}\end{array}$ & $\begin{array}{r}27 . \overline{0} \\
\overline{-} \\
33 . \overline{2}\end{array}$ & $\begin{array}{r}8.7 \\
9.1 \\
9.3 \\
10.7 \\
9.2\end{array}$ & $\begin{array}{l}29.5 \\
34.2 \\
35.7 \\
37.6 \\
36.2\end{array}$ \\
\hline $\begin{array}{l}\text { Jul } \\
\text { Aug } \\
\text { Sep } \\
\text { Oct } \\
\text { Nov } \\
\text { Dec }\end{array}$ & $\begin{array}{l}552.7 \\
552.9 \\
542.3 \\
570.9 \\
565.9 \\
580.2\end{array}$ & $\begin{array}{l}500.8 \\
480.8 \\
414.4 \\
409.1 \\
385.0 \\
457.3\end{array}$ & $\begin{array}{l}52.6 \\
44.4 \\
42.8 \\
55.6 \\
53.0 \\
38.1\end{array}$ & $\begin{array}{r}24.2 \\
-1.9 \\
-8.4 \\
-0.8 \\
4.0 \\
-5.8\end{array}$ & $\begin{array}{r}- \\
26 . \overline{0} \\
- \\
\overline{-} \\
28 . \overline{3}\end{array}$ & $\begin{array}{r}- \\
\overline{-} \\
19.8 \\
- \\
14 . \overline{4}\end{array}$ & $\begin{array}{l}8.8 \\
8.8 \\
9.2 \\
9.6 \\
8.9 \\
8.4\end{array}$ & $\begin{array}{l}32.6 \\
32.0 \\
28.4 \\
29.4 \\
26.2 \\
28.5\end{array}$ \\
\hline $\begin{array}{r}2009 \text { Jan } \\
\text { Feb } \\
\text { Mar } \\
\text { Apr } \\
\text { May }\end{array}$ & $\begin{array}{l}560.3 \\
511.6 \\
558.3 \\
541.1 \\
557.5\end{array}$ & $\begin{array}{l}462.1 \\
434.0 \\
497.5 \\
533.6 \\
563.0\end{array}$ & $\begin{array}{l}38.5 \\
34.4 \\
56.5 \\
57.5 \\
63.5\end{array}$ & $\begin{array}{l}30.1 \\
20.2 \\
26.1 \\
27.5 \\
33.4\end{array}$ & $\begin{array}{r}- \\
\overline{-} \\
26.0 \\
- \\
-\end{array}$ & $\begin{array}{r}- \\
\overline{-} \\
27.0 \\
- \\
-\end{array}$ & $\begin{array}{l}11.0 \\
10.9 \\
11.3 \\
11.0 \\
11.4\end{array}$ & $\begin{array}{l}29.8 \\
26.6 \\
33.3 \\
35.1 \\
36.4\end{array}$ \\
\hline
\end{tabular}

1 For further information refer to Section 6 of the Annual Supplement in the 4 Monthly figures are not available from 2006 onwards due to confidentiality isJanuary edition of Monthly Digest. sues.

2 Liquid milk that leaves the primary dairy in liquid form. This may be used for 5 Full cream, whole milk, partially skimmed milk and skimmed milk powders.

manufacture of other food products, or as liquid drinking milk.

3 Excludes production by direct sellers of liquid milk, butter and cheese. 
Monthly averages, calendar months or totals for four or five week periods; stocks: end of period

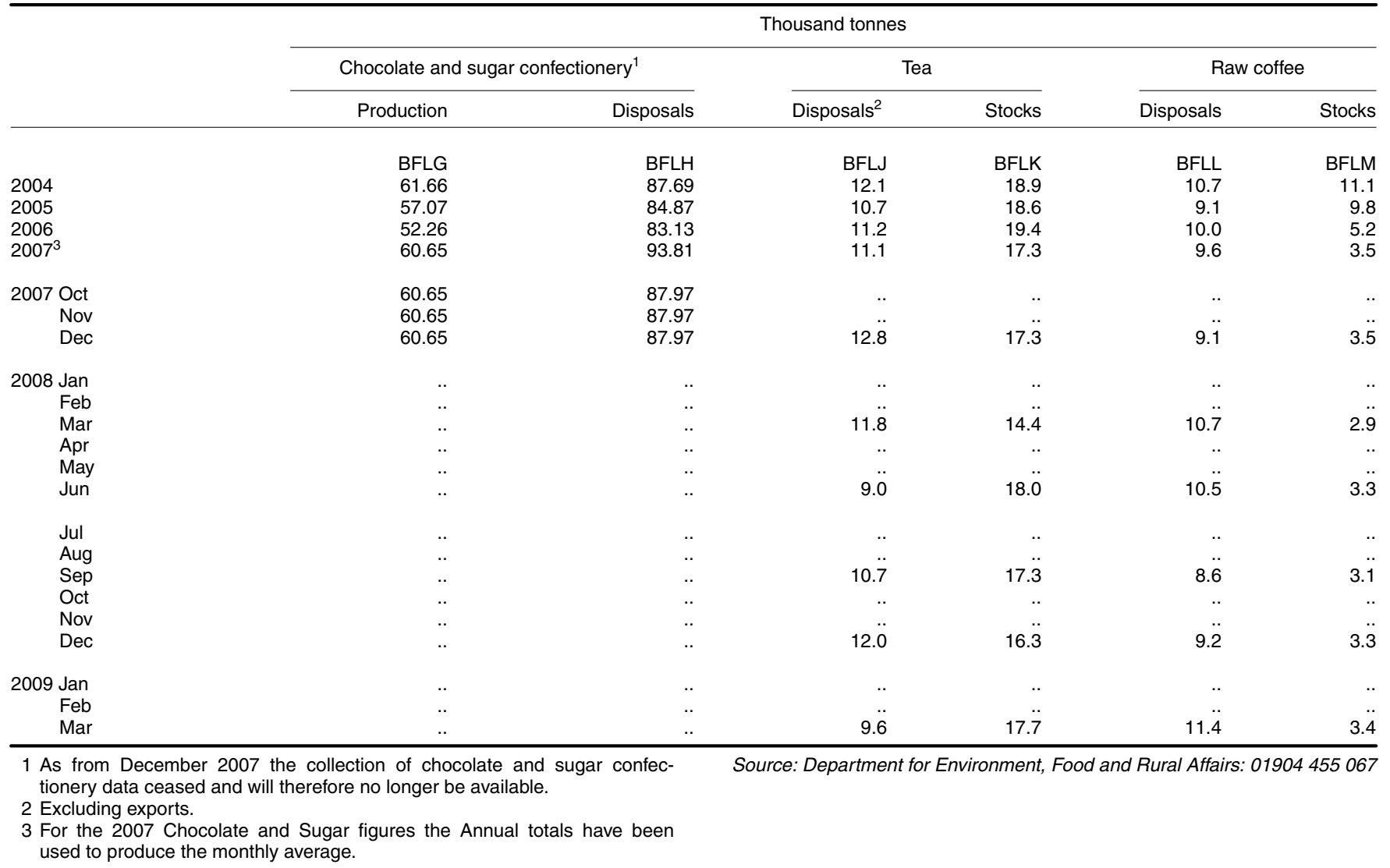

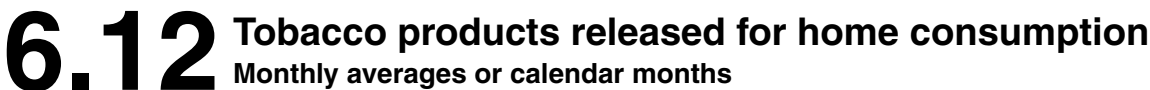

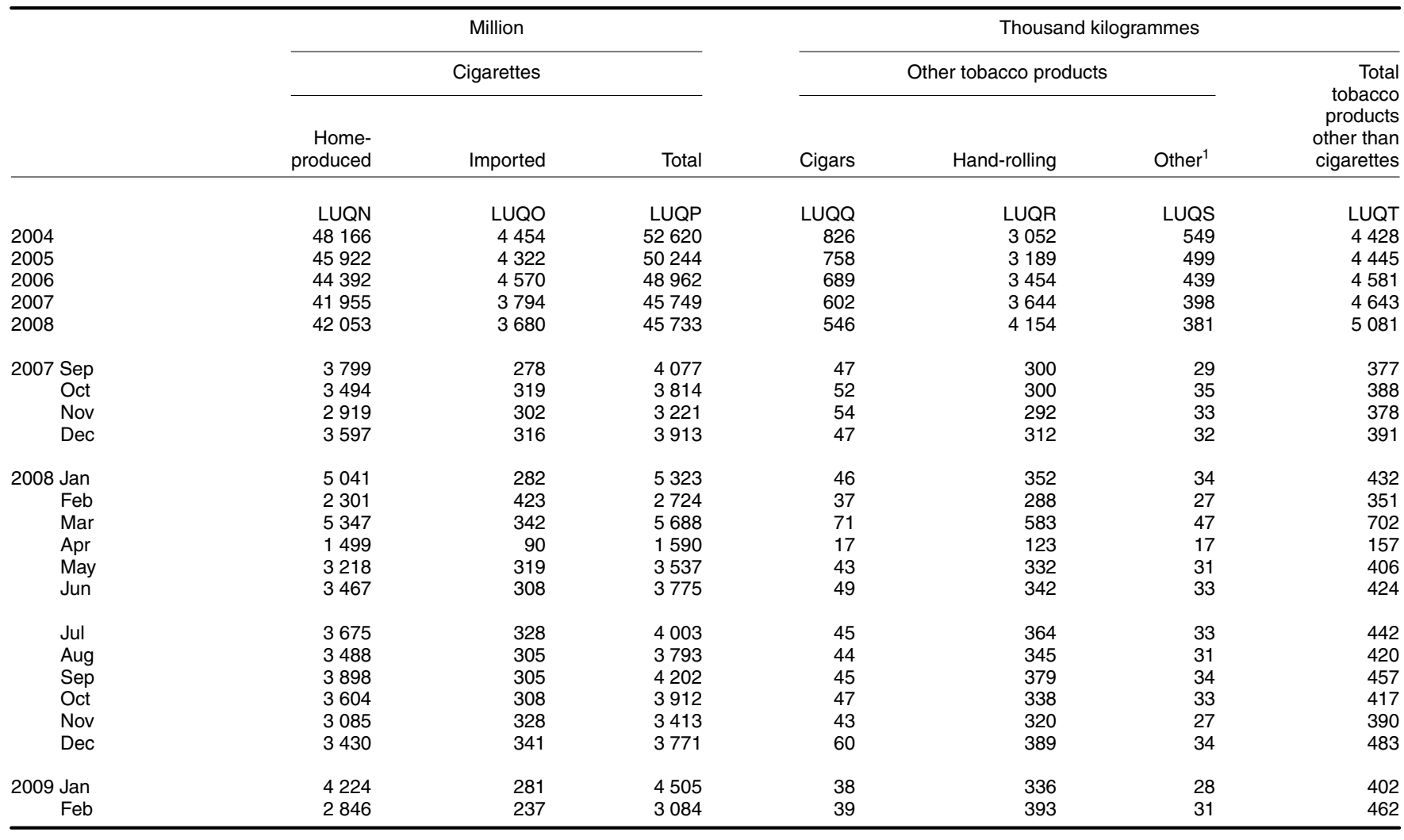




\section{$6.13^{\text {Alconolic drink }}$}

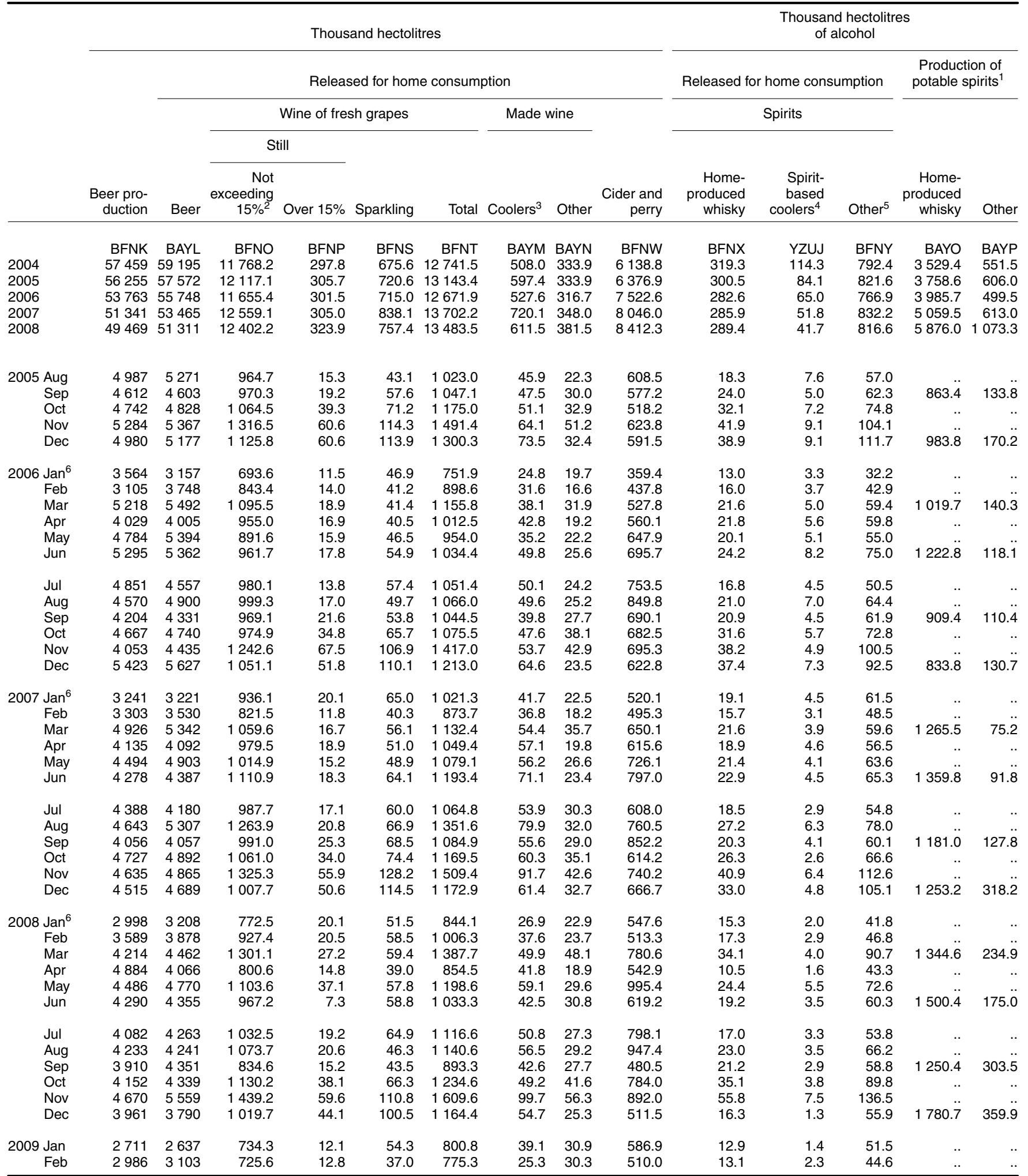

1 Data are available only quarterly and in the months following the quarter.

5 Includes imported spirits.

2 Percentage alcohol by volume.

6 Due to the effect of the holiday period, these figures are subject to greater un-

ade of appropriate strength.

4 From 28 April 2002 duty on spirit-based ready-to-drink (RTDs) products is charged at the same rate as spirits per litre of alcohol. Spirit-based RTDs were previously dutied at the made wine rate and details on quantities can certainty than usual. Also, unusually high or low figures may be changed on receipts of amendments to returns data.

be found in made wine coolers. 